\title{
Follow-up Case Report: Bumetanide Can Control Seizural Activity in Temporal Lobe Epilepsy Patient
}

\author{
Zeinab Gharaylou, ${ }^{1}$ Abbas Tafakhori, ${ }^{2}$ Masoumeh Rostampour, ${ }^{3}$ and Mahmoudreza Hadjighassem ${ }^{4,}$ \\ ${ }^{1}$ International Campus, Tehran University of Medical Sciences, Tehran, IR Iran \\ ${ }^{2}$ Iranian Center of Neurological Research, Tehran University of Medical Sciences, Tehran, IR Iran \\ ${ }^{3}$ Department of Medical Physics and Biomedical Engineering, Faculty of Medicine, Tehran University of Medical Sciences, Tehran, IR Iran \\ ${ }^{4}$ Brain and Spinal Cord Injury Research Center, Department of Neuroscience, School of Advanced Technologies in Medicine, International Campus, Tehran University of \\ Medical Sciences, Tehran. IR Iran \\ "Corresponding author: Mahmoudreza Hadjighassem, Brain and Spinal Cord Injury Research Center, Department of Neuroscience, School of Advanced Technologies in \\ Medicine, International Campus, Tehran University of Medical Sciences, Tehran, IR Iran. E-mail: mahmoudreza@hotmail.com
}

Received 2017 July 24; Accepted 2017 September 11.

\begin{abstract}
Despite the infectiveness of conventional anti-epileptic drugs (AEDs) in refractory focal epilepsy, surgery along with medications, as the only treatment, was not successful in 30\% of the cases. Recently, cysto- and histo-physiological investigations revealed the role of gamma-aminobutyric acid (GABA)-ergic system in etiopathology of temporal lobe epilepsy (TLE). Studies reported that the inhibitory action of GABA neurotransmitter due to elevated intracellular Cl-concentration through abnormal expression of Na-K-Cl cotransporter (NKCC)-1 became excessive in recurrent seizures. These primary results were confirmed in the brain tissue obtained from patients with TLE. Based on the evidence, the current study suggested the administration of bumetanide, an NKCC1 inhibitor, as a potential candidate for the treatment of intractable TLE. The previous study by authors showed a significant reduction in seizural activity of 2 out of 3 patients with TLE following the administration of bumetanide. However, result of the current study showed the control of seizure in the 3 rd case of previous study by the administration of bumetanide as an adjuvant to AEDs. Moreover, his AEDs dosage after discontinuation of Bumetanide tapered without any relapse.
\end{abstract}

Keywords: Head Injury, Oculomotor Palsy, Third Nerve

\section{Introduction}

Temporal lobe epilepsy (TLE) is one of the most prevalent forms of focal epilepsy, which is refractory to pharmacological treatment in $70 \%$ of the cases (1). Surgery is suggested for nonresponsive cases to reduce recurrence of seizures (2).Surgery in patients with refractory TLE by antiepileptic drugs (AEDs) is invasive, expensive, and carries a surgical risk; however, one-third of the patients develop seizural activity following surgery (3).

Bumetanide is a diuretic agent, which blocks $\mathrm{Na} / \mathrm{K} / \mathrm{Cl}$ cotransporter (NKCC1) (4). Experimental studies in neonatal animals showed that NKCC1 is highly expressed in neonatal period. Moreover, further studies revealed that brain insults resulting in recapitulation of immaturelike transmitter that were involved in epileptogenesis (5). Miles et al. reported that in hippocampal slices obtained from patients with TLE, interictal paroxysmal activity was generated by gamma-aminobutyric acid (GABA)-ergic depolarizing transmission in the pyramidal cells (4). Further studies revealed that shifting from inhibitory to excitatory of GABAergic responses was related to the intracellular chloride concentration produced by altered expression of $\mathrm{K} / \mathrm{Cl}$ cotransporter 2 (KCC2) and NKCC1 (4). Deidda et al. showed that bumetanide can reverse excitatory GABAA receptor by restoring Ecl to wild type and make synaptic plasticity and memory in a mouse model of Down syndrome (6). Moreover, bumetanide in combination with benzodiazepines was used to treat pharmacoresistant status epilepticus (7). Modification of chloride hemostasis by bumetanide may play a role in restoring neuronal functions.

Based on the above mentioned evidence, in a preliminary study authors showed that $2 \mathrm{mg} /$ day of bumetanide for 4 months effectively reduced seizure frequency in patients with TLE and resistant to drugs (8). Indeed, a recent study showed that $5 \mathrm{mg} /$ day of bumetanide for 2 months improved the Parkinson disease motor symptoms without any side effects (9).

The current paper reported the 3-year follow-up of 1 of the study subjects whose his seizure was controlled following the 4 months of adding bumetanide to his previous anti-epileptic regimen and AEDs tapering without any relapse. 


\section{Case Presentation}

The subject, a 36-year-old right handed male, was the 3rd patient in the authors" previous study (8). His first seizure occurred at the age of 24 years after dental surgery. He had no history of trauma, encephalopathy, hypoxia, diffuse, focal extratemporal electroencephalogram (EEG) spikes, or other cerebral lesions. The first seizure occurred at night, but he did not receive any treatment at that time. However, after the first attack, his epileptic seizure occurred. Complex-partial seizures started with 1-2 attacks monthly within a day. Six months later his seizure frequency increased to once a week. His seizure was initiated by loss of awareness followed by oral and right hand automatism and a short postictal phase.

\subsection{Diagnostic Focus and Assessment}

A long-term video-EEG monitoring confirmed the left anterior temporal lobe origin of the seizures and magnitude resonance imaging (MRI) (1.5T GE SIGNA LX) investigations showed left hippocampus and minimal right hippocampal sclerosis (Figure 1, left). Mild hypoperfusion at the left temporal lobe was detected in interictal single-photon emission computed tomography (SPECT). He received $900 \mathrm{mg}$ carbamazepine/day and depakine 500 $\mathrm{mg} /$ day. His seizures were not controlled even though with AEDs.

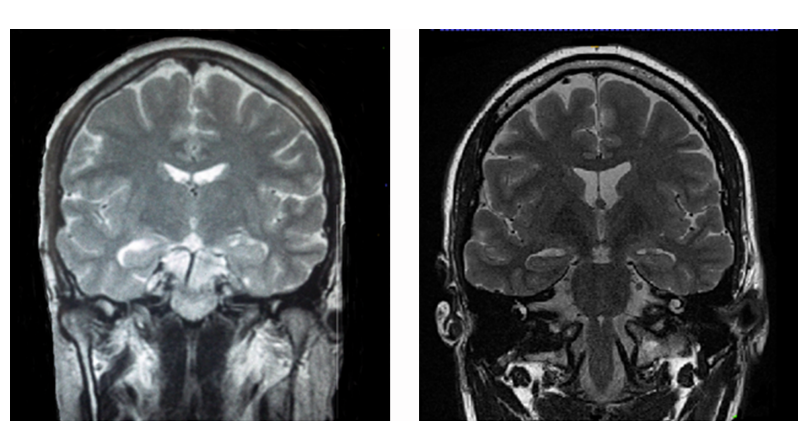

Figure 1. Video-EEG Monitoring

This patient was diagnosed with pharmacoresistant to 2 AEDs in adequate dosage. He was considered as a good candidate for left temporal lobectomy, but pre-surgical neurophysiological verbal and memory testing showed above-average risk.

\subsection{Therapeutic Focus and Assessment}

The case was enrolled in the study of receiving bumetanide twice a day ( $1 \mathrm{mg}$ every 12 hours) adjuvant to his AEDs for 4 months. Blood tests including uric acid, creatinine, serum $\mathrm{Na}+$ and $\mathrm{K}+$ were performed at the baseline (day 0), day 7, day 30, and day 90 after the administration of bumetanide. He was followed up 4 days before, during 4 months, and 3 years after the treatment. Long-term monitoring (LTM) (24 hours monitoring) was done using Stellate LTM System (Stellate, Montreal, QC, Canada) before and after the treatment (Figure $2 \mathrm{~A}$ and 2B) (8). A short EEG (30 minutes) was obtained recently (Figure 2C). In addition to the baseline MRI, brain MRI was performed according to a routine epilepsy protocol (10), using a 3.0-T MR imaging with a 32-channel coil (SYNGO MR B17, Trio Tim, SIEMENS) at the Neuroimaging Research Unit, Imam Khomeini Hospital, Tehran, Iran. The study included conventional MRI sequences: T1-mpreg., axial and coronal T2-weighted (Figure 1, right) and fluid attenuated inversion recovery. Seizure localization (on the 1st MRI) was analyzed on the 2nd MRI by 2 independent neuro-radiologists who confirmed decay of hypointensity of T1W images after treatment. To confirm the changes observed in the new images, binary hippocampal volume of patient was automatically analyzed using Free Surfer software version 5.3 (https://surfer.nmr.mgh.harvard.edu) in comparison with 10 healthy age and gender matched controls [mean age \pm standard deviation (SD): $35.7 \pm 0.67$ years] (Table 1 ).

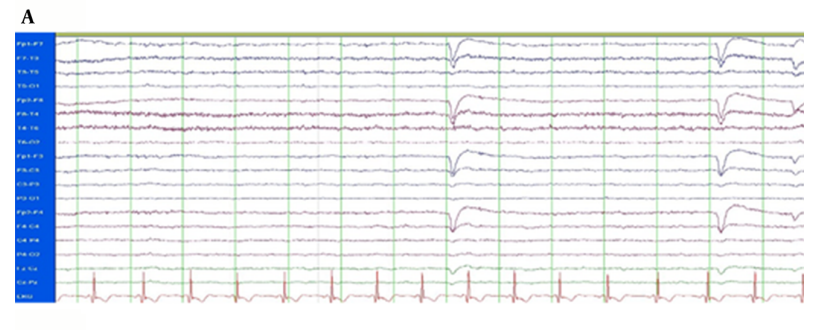

B

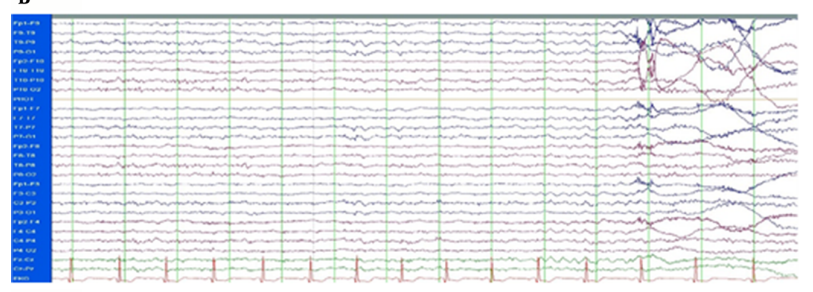

C

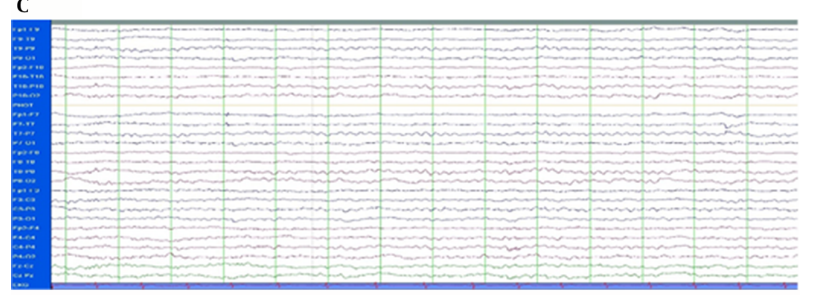

Figure 2. Long-Term Monitoring 
Table 1. Comparison of Hippocampal and Amygdala Volume Between the Patient and Healthy Controls

\begin{tabular}{|c|c|c|c|c|c|}
\hline Variables & \multicolumn{2}{|c|}{ Healthy Group } & \multicolumn{2}{|c|}{ Patient } & P Value Between Groups (Independent T Test) \\
\hline Volume & \multicolumn{2}{|c|}{ Hippocampal } & \multicolumn{2}{|c|}{ Hippocampal } & \\
\hline Side & Right & Left & Right & Left & Right Hippocampal: 0.33 \\
\hline Value & 78 & 77.89 & 76.52 & 75.22 & Left Hippocampal: 0.58 \\
\hline Volume & \multicolumn{2}{|c|}{ Amygdala } & \multicolumn{2}{|c|}{ Amygdala } & \\
\hline Side & Right & Left & Right & Left & Right Amygdala: 0.36 \\
\hline Value & 78.69 & 79.60 & 77.36 & 76.85 & Left Amygdala: 0.56 \\
\hline
\end{tabular}

Statistical package for social sciences (SPSS $®$, SPSS Inc., and Chicago, IL, USA) version 20 was used for the statistical data analysis. The independent $t$ test was used to analyze the differences between the case and healthy controls. Differences between the left and right hippocampal volumes were compared using the paired t test. P value of 0.05 was considered significant. The volumetric study showed reduction in the volume of hippocampus and amygdala in patient, but it was not statistically significant compared with those of the healthy ones $(\mathrm{P} \leq 0.5)$.

\subsection{Follow-Up and Outcomes}

The 3-year follow-up showed seizure frequency reduction $\geq 75 \%$ four months after discontinuation of bumetanide, while he was on carbamazepine (900 $\mathrm{mg} /$ day) and depakine (500 mg/day). The patient had noted 3 complex-partial seizure activities after very stressful situations, but was seizure free from the last one up to now. He had residual auras for 2 years, but from a year ago he was free from aura. He was regularly visited by a neurologist for 3 years. Dosage of his AEDs was not changed within the 1st year after cessation of bumetanide. Since 2 years ago, his AEDs dosage was tapered. Now he is on $400 \mathrm{mg}$ carbamazepine/day and depakine $200 \mathrm{mg} /$ day without any relapse.

\section{Discussion}

Bumetanide is recently considered as a new anticonvulsant therapy in neonates (11), schizophrenia (12), and neurodevelopmental disorders (13). It may revert excitation mediated by GABA through modifying chloride homeostasis. It suppresses GABA-generated network activities that contribute to the rhythmic interictal events $(14,15)$. Interictal events were not detected in the current case after bumetanide treatment. For the 1st time, the current report showed achievement of sustains shutting down of seizural activity after adding bumetanide to EAD's regimen. The current case report supported the possible long-term effect of bumetanide on refractory TLE without any side effects, while it was used for 4 months. Another study highlighted the long-term effect of this medication on sensory behaviors in a case study (16). It is proposed that rather than direct effects on NKCC1 and chloride homeostasis, bumetanide may increase the efficacy of postsynaptic inhibition of other AEDs and thereby act as anticonvulsant drugs. Investigation of bumetanide as an adjunctive anticonvulsant on adults undoubtedly requires more participants. Authors are conducting a new study hiring more patients with more frequent seizures. A preliminary observation was quite promising (unpublished data).

\section{References}

1. Goldenberg MM. Overview of drugs used for epilepsy and seizures: etiology, diagnosis, and treatment. P T. 2010;35(7):392-415. [PubMed: 20689626].

2. Jardim AP, Neves RS, Caboclo LO, Lancellotti CL, Marinho MM, Centeno RS, et al. Temporal lobe epilepsy with mesial temporal sclerosis: hippocampal neuronal loss as a predictor of surgical outcome. Arq Neuropsiquiatr. 2012;70(5):319-24. [PubMed: 22618783].

3. Thom M, Liagkouras I, Elliot KJ, Martinian L, Harkness W, McEvoy A, et al. Reliability of patterns of hippocampal sclerosis as predictors of postsurgical outcome. Epilepsia. 2010;51(9):1801-8. doi: 10.1111/j.15281167.2010.02681.x. [PubMed: 20738385].

4. Loscher W, Puskarjov M, Kaila K. Cation-chloride cotransporters NKCC1 and KCC2 as potential targets for novel antiepileptic and antiepileptogenic treatments. Neuropharmacology. 2013;69:62-74. doi: 10.1016/j.neuropharm.2012.05.045. [PubMed: 22705273].

5. Marguet SL, Le-Schulte VT, Merseburg A, Neu A, Eichler R, Jakovcevski I, et al. Treatment during a vulnerable developmental period rescues a genetic epilepsy. Nat Med. 2015;21(12):1436-44. doi: 10.1038/nm.3987. [PubMed: 26594844].

6. Deidda G, Parrini M, Naskar S, Bozarth IF, Contestabile A, Cancedda L. Reversing excitatory GABAAR signaling restores synaptic plasticity and memory in a mouse model of Down syndrome. Nat Med. 2015;21(4):318-26. doi: 10.1038/nm.3827. [PubMed: 25774849].

7. Sivakumaran S, Maguire J. Bumetanide reduces seizure progression and the development of pharmacoresistant status epilepticus. Epilepsia. 2016;57(2):222-32. doi: 10.1111/epi.13270. [PubMed: 26659482].

8. Eftekhari S, Mehvari Habibabadi J, Najafi Ziarani M, Hashemi Fesharaki SS, Gharakhani M, Mostafavi $\mathrm{H}$, et al. Bumetanide reduces seizure frequency in patients with temporal lobe epilepsy. Epilep- 
sia. 2013;54(1):e9-12. doi: 10.1111/j.1528-1167.2012.03654.x. [PubMed: 23061490].

9. Damier P, Hammond C, Ben-Ari Y. Bumetanide to Treat Parkinson Disease: A Report of 4 Cases. Clin Neuropharmacol. 2016;39(1):57-9. doi: 10.1097/WNF.0000000000000114. [PubMed: 26757306].

10. Vasta R, Caligiuri ME, Labate A, Cherubini A, Mumoli L, Ferlazzo E, et al. 3-T magnetic resonance imaging simultaneous automated multimodal approach improves detection of ambiguous visual hippocampal sclerosis. Eur J Neurol. 2015;22(4):725-e47. doi: 10.1111/ene.12648. [PubMed: 25598219].

11. Kahle KT, Staley KJ. The bumetanide-sensitive Na-K-2Cl cotransporter NKCC1 as a potential target of a novel mechanism-based treatment strategy for neonatal seizures. ; 2008.

12. Lemonnier E, Lazartigues A, Ben-Ari Y. Treating Schizophrenia With the Diuretic Bumetanide: A Case Report. Clin Neuropharmacol. 2016;39(2):115-7. doi: 10.1097/WNF.0000000000000136. [PubMed:
26966887]

13. Bruining H, Passtoors L, Goriounova N, Jansen F, Hakvoort B, de Jonge $\mathrm{M}$, et al. Paradoxical Benzodiazepine Response: A Rationale for Bumetanide in Neurodevelopmental Disorders? Pediatrics 2015;136(2):e539-43. doi: 10.1542/peds.2014-4133. [PubMed: 26216321].

14. Huberfeld G, Wittner L, Clemenceau S, Baulac M, Kaila K, Miles R, et al. Perturbed chloride homeostasis and GABAergic signaling in human temporal lobe epilepsy. J Neurosci. 2007;27(37):9866-73. doi: 10.1523/JNEUROSCI.2761-07.2007. [PubMed: 17855601].

15. Miles R. Chloride homeostasis and GABA signaling in temporal lobe epilepsy. ; 2012

16. Grandgeorge M, Lemonnier E, Degrez C, Jallot N. The effect of bumetanide treatment on the sensory behaviours of a young girl with Asperger syndrome. BMJ Case Rep. 2014;2014 doi: 10.1136/bcr2013-202092. [PubMed: 24488662]. 